http://dx.doi.org/10.35381/e.k.v4i7.1269

\title{
Toxicología de los materiales pictóricos y riesgos sanitarios del artista
}

Toxicology of pictorial materials and health risks of the artist

\author{
Carmen Elena Carbonell-García \\ caelcg@hotmail.com \\ Escuela Superior de Bellas Artes Macedonio de la Torre, Trujillo \\ Universidad César Vallejo, Lima \\ Perú \\ https://orcid.org/0000-0002-3692-3013 \\ Rufino Rodríguez-Román \\ rufinorodriguezr30@gmail.com \\ Universidad César Vallejo, Lima \\ Perú \\ https://orcid.org/0000-0002-5470-9918 \\ Juan Aarón Rodríguez-Carbonell \\ aaronroca@hotmail.com \\ Universidad Antenor Orrego, Trujillo \\ Perú \\ https://orcid.org/0000-0001-9490-5262
}

Recepción: 20 de marzo 2020

Revisado: 15 de abril 2020

Aprobación: 22 de mayo 2021

Publicación: 01 de junio 2021 


\title{
RESUMEN
}

La actual investigación tiene por objetivo analizar la toxicología de los materiales pictóricos y riesgos sanitarios del artista plástico del Perú. El proceso metodológico fue desarrollado desde un tipo de investigación documental con diseño bibliográfico, teniéndose como muestra poblacional 10 publicaciones relacionadas al tema con la finalidad de conocer los riesgos toxicológicos a los cuales se ven expuestos los artistas plásticos peruanos al plasmar sus obras para el beneplácito de los espectadores. La toxicidad de los materiales que se pueden emplear en la obra, entre los cuales se encuentran mercurio, plomo, arsénico, entre otros, desconocidos al momento de emplear el material que pueden ocasionar graves daños a la salud como sucedió con el síndrome de Ardystil, lo cual fue contraproducente para la salud humana, siendo necesario promover la concienciación tanto del artista como a los fabricantes para evitar acciones perjudiciales contra la vida humana y ambiental.

Descriptores: Toxicología; contaminante; pintor. (Palabras tomadas del Tesauro UNESCO).

\begin{abstract}
The current research aims to analyze the toxicology of pictorial materials and health risks of the plastic artist from Peru. The methodological process was developed from a type of documentary research with bibliographic design, having as a population sample 10 publications related to the subject in order to know the toxicological risks to which Peruvian plastic artists are exposed when they translate their works for the approval of the spectators. The toxicity of the materials that can be used in the work, among which are mercury, lead, arsenic, among others, unknown at the time of using the material that can cause serious damage to health as happened with Ardystil syndrome, which was counterproductive for human health, making it necessary to promote the awareness of both the artist and the manufacturers to avoid harmful actions against human and environmental life.
\end{abstract}

Descriptors: Toxicology; pollutants; painters. (Words taken from the UNESCO Thesaurus). 


\section{INTRODUCCIÓN}

Al apreciar una obra de arte, se percibe la estética plasmada por el o la artista que lo ha diseñado, lo cual, a la vista del espectador se presenta una figura sensible e ingenua, pero que guarda en sí misma, residuos tóxicos que pudieron atentar contra la salud del pintor, tal como comentan, (Camacho, et al. 2011), al deducir como posible causa de muerte de "Vermeer", el consumo de plomo y mercurio, contentivo en los materiales plásticos que empleaba, al tener contacto con la boca y piel, al manipularlos ingenuamente sin imaginarse la contaminación generada sobre su organismo.

Además, el mercurio y plomo, como metales pesados no solo son contraproducentes para la salud humana, animal, sino, contaminante del medio ambiente, causando en el caso de las personas, intoxicación que pueden originar cáncer, e incluso la muerte (Londoño-Franco, et al. 2016). Por otro lado, esta visión es compartida por (Tomailla \& lannacone, 2018), al indicar que el "arsénico, cadmio, mercurio y plomo"; generan severas contaminaciones al medio ambiente, peces, cuando se vierte en altas concentraciones, lo que permite generar sensibilidad sobre el riesgo que corren los artistas plásticos, al trabajar con materiales contentivos de materiales tóxicos.

En razón de lo expuesto, la actual investigación tiene por objetivo analizar la toxicología de los materiales pictóricos y riesgos sanitarios del artista plástico del Perú.

\section{METODOLOGÍA}

El proceso metodológico fue desarrollado desde un tipo de investigación documental con diseño bibliográfico, teniéndose como muestra poblacional 10 publicaciones relacionadas al tema con la finalidad de conocer los riesgos toxicológicos a los cuales se ven expuestos los artistas plásticos peruanos al plasmar sus obras para el beneplácito de los espectadores; trabajo que permite además, sensibilizar tanto a los artistas como a los usuarios, a tener en cuenta el potencial contaminante contentivo en una obra de arte. Para estructurar un corpus en contribución del estado de la cuestión sobre el tema, se 
presenta una síntesis teórica a partir del método analítico - sintético, el cual fue implementado para gestionar desde argumentación analítica de los investigadores, una proposición como síntesis del proceso investigativo a la luz de aplicar la técnica de análisis de contenido a cada documento escrutado como muestra poblacional.

\section{RESULTADOS}

En esta sección, se describen los resultados escrutados a fin de construir la síntesis teórica como aporte del estado de la cuestión del tema investigado:

En primer orden, (Doubrovskaia, 2019), comenta sobre la incertidumbre si realmente el arte está produciendo contaminación el ser humano y medio ambiente, destacando que aunque no existen investigaciones conclusivas para responder con certeza tal interrogante, es conocida la existencia de materiales pesados y tóxicos en la gran mayoría de los materiales empleados por los artistas, lo cual, a simple deducción conlleva a responder afirmativamente sobre la alta posibilidad de contaminación que se corre, hasta tal punto, que exhorta evitar realizar arte a partir de los siguientes materiales:

a) Pintura con base de aceite, pues puede contener pigmentos tóxicos como plomo, manganeso, mercurio, cadmio, etc. Lo mismo ocurre con la pintura a base de agua como el acrílico, la acuarela y la témpera. Cuando utilices pinturas a base de aceite, evita los disolventes y diluyentes como los alcoholes minerales y la trementina.

b) Los pasteles pueden contener plomo, cadmio, cromo y otros compuestos tóxicos. Los pasteles desprenden mucho polvo y requieren un fijador igualmente tóxico para fijar el dibujo al papel. Cuando se trabaja con pasteles, lo mejor es usar guantes y una máscara.

c) Los barnices y lacas están hechos con resinas disueltas en solventes y son altamente tóxicos. 
d) Materiales de aerografía y pintura en aerosol, pues contienen pigmentos y disolventes tóxicos y son especialmente peligrosos en espacios con poca ventilación.

e) Solventes químicos, como el alcohol metílico y el tolueno, que se utilizan en esterilizaciones y fijadores, algunos marcadores permanentes, medios y barnices, tintas para serigrafía, bases de grabado, cemento de caucho y algunos otros adhesivos, esmaltes, lacas y trementina. En los suministros de pintura, disuelven los pigmentos -algunos de los cuales también son tóxicos- y permiten que la pintura se extienda uniformemente. Estas sustancias representan un grave peligro para la salud, ya que causan daños a los órganos y provocan ceguera en caso de ingestión.

f) Ciertos esmaltes para cerámica, pues a menudo contienen metales pesados como cadmio, cromo y plomo. La cocción en horno libera más químicos tóxicos a la atmósfera, dañando el medio ambiente además de perjudicar tu salud. Además, la arcilla seca contiene polvo de sílice y también puede contener talco y asbesto. Asegúrate de que tu estudio esté bien ventilado y utiliza arcilla húmeda

g) Yesos, pues los yesos pueden crear mucho polvo tóxico. Lee la etiqueta y compra las versiones más seguras de este material.

h) Los marcadores aromáticos a base de disolventes contienen xileno, una sustancia química muy peligrosa para el sistema nervioso, los riñones y los sistemas respiratorio y reproductivo. Los marcadores a base de agua generalmente no representan un grave peligro para la salud.

i) Las tintas de impresión (a base de agua o aceite) a menudo usan pigmentos tóxicos que contienen compuestos inorgánicos peligrosos. Algunas tintas contienen disolventes y conservantes. El proceso de impresión también incluye ácidos como el ácido fluorhídrico, que es muy tóxico. Un estudio de grabado, 
litografía o serigrafía puede estar lleno de vapores dañinos. Si tienes que trabajar con estos medios, iponte una máscara!

j) Cemento de caucho y pegamento para modelos, sustancias altamente tóxicas, ciertamente no son apropiadas para que las utilicen los niños. El cemento de goma contiene una neurotoxina denominada hexano.

La autora destaca como medida preventiva, la necesidad que tiene el artista plástico de conocer la toxicidad de los materiales con los cuales trabaja, en este sentido, señala que por ejemplo en los Estados Unidos, se debe realizar arte a partir de los productos etiquetados bajo la norma ASTM D 4236, aunque considera que no es suficiente por cuanto, no describe detalladamente si tales materiales contienen tóxicos y la cantidad en la cual, lo poseen, contribuyendo en indicar la necesidad de trabajar en talleres donde la ventilación natural, sea sustancial con la finalidad de contribuir en la salud del artista, así mismo, invita a considerar el adecuado manejo para la preservación del medio ambiente. Condición corroborada por (Sacristán-Cuadrón, 2004), al indicar la necesidad de contar con ventilación suficiente para minimizar el grado de contaminación o toxicidad en el artista, sobre todo cuando los materiales aerográficos pueden contener ingredientes nocivos para la salud, entre los cuales se encuentra el antecedente conocido como el síndrome de Ardystil; el cual causó revuelo en España en 1992, siendo catalogada como una enfermedad poco frecuente, asociada en inhalación en ambiente laboral, provocando alternaciones en el sistema nervioso central y neuropatía (Cordero \& Solé, 1995). (Nemery \& Hoet, 2015), comentan que estos brotes recientes de enfermedades respiratorias es que los productos químicos nunca deben considerarse inofensivos para los pulmones a menos que esto se haya demostrado mediante pruebas adecuadas de toxicidad pulmonar; por cuanto esto puede afectar no solo al artista, sino, a su cuadro familiar, propiciando que a partir de la experiencia de síndrome de Ardystil, (Reig-Botella \& Clemente-Díaz, 2010), presentan resultados de cuadros depresivos en el trabajador a causa de haber sido afectados por la enfermedad, además de perder calidad de vida 
laboral, considerándose necesario la prevención por parte de las empresas en favor de preservar la salud como medida motivadora que proyecte innovación y liderazgo en los trabajadores.

La afección pulmonar por contaminantes en los aerosoles, puede desencadenar en la salud del afectado, un camino incierto de recuperación, (Candela, 2012) a veinte años de la tragedia por el síndrome de Ardystil, indicaba que aun padecían problemas pulmonares y derivados, lo cual indica que han tenido que vivir durante largo tiempo con secuelas de un producto que en teoría debía ser inofensivo y ser empleado con fines artísticos, lo cual delata que los fabricantes pueden introducir sustancias sin tener en cuenta la sanidad humana - ambiental.

La contaminación ambiental juega un papel preponderante en la salud humana y de las especies vivas, viéndose afectado por cargas de sustancias como el arsénico (RangelMontoya, et al. 2015), siendo corroborada por (Díaz-Arenas \& Silva-Jiménez, 2018), al indicar sobre la necesidad de promover preservación del medio ambiente a la luz de los metales pesados que son lanzados al mismo, siendo que los materiales contentivos en algunos materiales empleados por los artistas plásticos, pueden estar en el orden contaminante del ser humano y del ambiente.

Entre los contaminantes más poderosos a los cuales se enfrenta el artista plástico es el mercurio, (María, 2017), destaca las afecciones que pueden sobrevenir por intoxicación con este metal pesado, lo cual, para el contexto del artista plástico, se corresponde con los planteamientos de (Camacho, et al. 2011), (Londoño-Franco, et al. 2016) y (Tomailla \& lannacone, 2018); en donde se señala el grave peligro para la salud humana el consumo de elementos tóxicos durante el ejercicio artístico.

El mercurio tiene efectos sobre el sistema nervioso central (Ramírez, 2008), lo cual, puede introducirse silenciosamente en la salud del artista, por lo que (Méndez-Visag, 2014), indica que se debe manejar con responsabilidad el mercurio en favor de prevenir adversidades sanitarias, sobre todo cuando (BBC News Mundo, 2019), como la escultora 
Gillian Gensen, se estaba envenenando silenciosamente al crear la escultura llamada Adán.

\section{CONCLUSIÓN}

El arte plástico se encuentra ligado no solo a la estética o al metalenguaje que pretende emitir, se ve envuelto también en la toxicidad de los materiales que se pueden emplear en la obra, entre los cuales se encuentran mercurio, plomo, arsénico, entre otros, desconocidos al momento de emplear el material que pueden ocasionar graves daños a la salud como sucedió con el síndrome de Ardystil, esto invita a reflexionar sobre tomar medidas de prevención sobre el valor de articular la salud en el artista plástico, se hace necesario promover una educación artista ambiental con la finalidad no solo de exhortar al artista, sino, también a los fabricantes para no involucrar altos grados de toxicidad en los productos que expenden.

\section{FINANCIAMIENTO}

No monetario.

\section{AGRADECIMIENTO}

A todos los agentes sociales involucrados; por motivar el desarrollo de la Investigación.

\section{REFERENCIAS CONSULTADAS}

BBC News Mundo (2019). Cómo una escultura envenenó poco a poco a su creadora [How a sculpture little by little poisoned its creator]. Recuperado desde https://www.bbc.com/mundo/noticias-46850987

Camacho, T, Piñero, MF \& Pallas, E. (2011). Vermeer: Los colores tóxicos del plomo y del mercurio [Vermeer: The Toxic Colors of Lead and Mercury]. Rev. Toxicol, 28, 115-118. https://n9.cl/0ut5n 
EPISTEME KOINONIA

Revista Electrónica de Ciencias de la Educación, Humanidades, Artes y Bellas Artes

Año IV. Vol IV. Nº7. Enero - Junio. 2021

Hecho el depósito de Ley: FA2018000022

ISSN: 2665-0282

FUNDACIÓN KOINONIA (F.K)

Santa Ana de Coro, Venezuela

Carmen Elena Carbonell-García; Rufino Rodríguez-Román; Juan Aarón Rodríguez-Carbonell

Candela, M. (2012). El drama interminable de Ardystil [The endless drama of Ardystil]. Recuperado desde https://n9.cl/1uciy

Cordero, P., \& Solé, A. (1995). Bronquiolitis obliterante con neumonía organizada (síndrome Ardystil) y enfermedad desmielinizante crónica del sistema nervioso central [Bronchiolitis obliterans organizing pneumonia (Ardystil syndrome) and chronic demyelinating disease of the central nervous system]. Archivos de bronconeumologia, 31(2), 89-92. https://doi.org/10.1016/s0300-2896(15)30971-6

Díaz-Arenas, J. \& Silva-Jiménez, K. (2018). Identificación de niveles de toxicidad de metales pesados en el suelo y los potenciales peligros a la salud humana [Identification of heavy metal toxicity levels in the soil and potential human health hazards]. Revista Dinámica Ambiental, 2(2), 1-11.

Doubrovskaia, M. (2019). Apuesta por la ecología: Prácticas respetuosas con el medio ambiente para artistas. He aquí cómo lograr que tu trabajo artístico sea lo más ecológico posible [Going Green: Environmentally Friendly Practices for Artists. Here's how to make your artwork as eco-friendly as possible]. Recuperado desde https://n9.cl/0enby

Londoño-Franco, L, Londoño-Muñoz, P, \& Muñoz-García, F. (2016). Los riesgos de los metales pesados en la salud humana y animal [Risk of heavy metals in human and animal health]. Biotecnología en el Sector Agropecuario y Agroindustrial, 14(2), 145-153.

María L. (2017). Taki Onqoy: epidemia de intoxicación por exposición al mercurio en Huamanga del siglo XVI [Taki Onqoy: mercury poisoning epidemic in 16TH century Huamanga]. Revista peruana de medicina experimental y salud pública, 34(2), 337-342. https://doi.org/10.17843/rpmesp.2017.342.2738

Méndez-Visag C. (2014). Manejo responsable del mercurio de la amalgama dental: una revisión sobre sus repercusiones en la salud [Responsible management of dental amalgam mercury: a review of its impact on health]. Revista peruana de medicina experimental y salud publica, 31(4), 725-732.

Nemery, B., \& Hoet, P. H. (2015). Humidifier disinfectant-associated interstitial lung disease and the Ardystil syndrome. American journal of respiratory and critical care medicine, 191(1), 116-117. https://doi.org/10.1164/rccm.201409-1726LE 
Parachaeirodon innesi neon tetra (Characidae)]. Rev. Toxicol, 35, 95 - 105

Ramírez, V. (2008). Intoxicación ocupacional por mercurio [Mercury occupational poisoning]. Anales de la Facultad de Medicina, 69(1), 46-51.

Rangel-Montoya, E, Montañez-Hernández, L., Luévanos Escareño, P., \& Balagurusamy, N. (2015). Impacto del arsénico en el ambiente y su transformación por microorganismos [Impact of arsenic on the environment and its microbial transformation]. Terra Latinoamericana, 33(2), 103-118.

Reig-Botella, A., \& Clemente-Díaz, M. (2010). Análisis del área sociofamiliar y laboral en las personas afectadas por el síndrome de Ardystil [Analysis of the social, family and work area in people affected by Ardystil syndrome]. Barataria. Revista Castellano-Manchega De Ciencias Sociales, (11), 171-185. https://doi.org/10.20932/barataria.v0i11.158

Sacristán-Cuadrón, R. (2004). Toxicología de los Materiales Pictóricos [Pictorial Materials Toxicology]. Recuperado desde https://eprints.ucm.es/id/eprint/1752/1/T23415.pdf

Tomailla, J., \& lannacone, J. (2018). Toxicidad letal y subletal del arsénico, cadmio, mercurio y plomo sobre el pez Parachaeirodon innesi neon tetra (Characidae) [Lethal and sublethal toxicity of arsenic, cadmium, mercury and lead on fish 